\title{
La source de l'étonnement
}

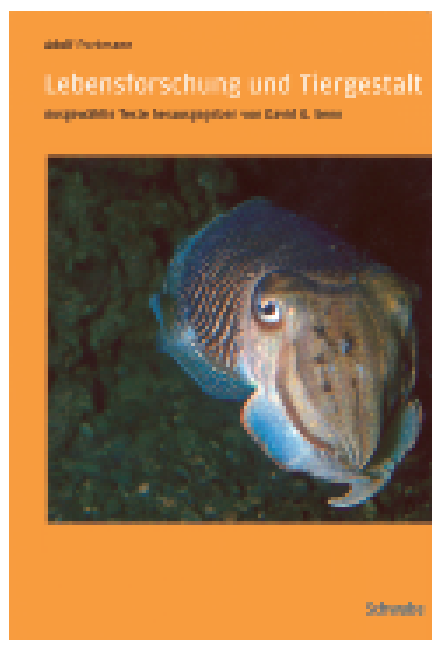

* A. Portmann

Lebensforschung und Tiergestalt. Ausgewählte Texte, herausgegeben von David G. Senn.

Basel: Schwabe; 2006. $231 \mathrm{~S}$.

ISBN 3-7965-2172-X. 48
Sur un ferry-boat gigantesque en Méditerranée, il peut arriver qu'accoudé au bastingage à 20 mètres au-dessus de l'eau et exposé à des rafales tempétueuses, on vive à l'improviste un événement source d'intense étonnement. Voici comment cela fonctionne: on serre un bout de pain de la grosseur d'un morceau de sucre entre le pouce et l'index de la main droite, par exemple - suivant que l'on se trouve à tribord ou à bâbord -, on tend tranquillement le bras par-dessus la rambarde en position d'abduction à $90^{\circ}$ (ou un peu davantage), et l'on attend ...

Et le voici qui ne tarde pas: un goéland vient au lof, planant en position légèrement oblique, et se place à cinq mètres environ derrière la main qui lui est tendue.

Non, mon ami, tu n'y arriveras jamais, pense-t-on devant les fortes bourrasques qui, avec une irrégularité perfide, déstabilisent l'élégant volatile et, tels les coups bien minutés d'un boxeur, le font dériver en l'éloignant non de cinq, mais de dix mètres. Pour compliquer les choses et éviter tout ennui, un trou d'air se forme soudain, ou alors, sans prévenir, l'oiseau se voit catapulté en hauteur. Notre nouvel ami reste serein, il se rapproche peu à peu de la bouchée convoitée. Encore un mètre, mais voici qu'une rafale le jette de côté. Encore $50 \mathrm{~cm}$, mais voilà que l'air semble céder sous lui et qu'il se retrouve deux étages plus bas. Et pourtant, comme on vient de le pressentir, le moment miracle où tout fonctionne est arrivé: comme un surfeur sur la vague, le goéland, en vol plané, glisse obliquement en profondeur, se joue de quelques petites turbulences et, virant majestueusement vers le large, saisit en passant la récompense de son coup de maître.

Voilà une expérience qui réveille en nous des souvenirs enfouis depuis longtemps dans le tréfonds de la mémoire. Par exemple de ce professeur de neuroinformatique qui, pince sans rire, releva un jour au cours d'une conversation, de façon plutôt inattendue, qu'en comparaison avec une abeille qui prend possession de «sa» fleur puis s'en régale, un ordinateur super moderne est incroyablement stupide.

L'art de ce goéland nous rappelle aussi, et de manière encore plus vive, certaines phrases du remarquable ouvrage «Lebensforschung und Tiergestalt», qui contient des textes choisis du grand biologiste bâlois Adolf Portmann $(1897-1982)^{*}$. Des assertions comme celle-ci (traductions FMH): «Lorsqu'on s'est efforcé pen- dant des décennies d'approfondir notre compréhension des rapports entre forme et fonction en partant de l'exemple des plumes de l'oiseau, comme nous l'avons fait dans mon laboratoire, on se trouve tout de même régulièrement confronté à des créations qui échappent à tout essai d'interprétation». Ou comme celle-ci: «Le pressentiment que nous avons de l'insondable étendue de la réalité qui nous entoure et qui, entité gigantesque, englobe tout notre être, ce pressentiment nous pousse à percevoir l'imperfection de notre vision du monde, telle que nous la façonnons par la raison, et à sans cesse tenter de la compléter en imaginant les rapports cachés entre les choses».

Portmann n'était pas un rêveur dépourvu d'esprit scientifique, bien au contraire. C'est par des travaux de biométrie très exacts sur l'évolution et la croissance des vertébrés supérieurs et notamment sur le développement de leur cerveau - qu'il s'est acquis une réputation internationale. Mais il ne s'est pas limité aux manifestations du vivant qui se coulent parfaitement et sans contradiction dans le canon des structures modèles établies. Sa curiosité a été éveillée par des phénomènes qui échappent aux explications biologiques et fonctionnelles courantes. Une caractéristique qu'il partage d'ailleurs avec de nombreux grands noms de l'histoire de la science. Récemment encore, le zoologiste Günter Tembrock, lui aussi un ténor en la matière, citait, dans un entretien à la Weltwoche, l'exemple - énigmatique en termes de théorie de l'évolution - d'un type de chouette qui vit dans une caverne et effraie ses attaquants potentiels en imitant le son du serpent à sonnettes: «Un thème passionnant et un défi pour la biologie de l'évolution. Il est extrêmement difficile d'imaginer comment, par simple sélection, le cri d'une chouette a pu devenir celui d'un serpent à sonnettes.»

En fin de compte, la science ne peut progresser qu'en regardant de telles questions en face. C'est dans ces zones limites qui sont source d'étonnement, là où la théorie ne permet plus d'expliquer de manière satisfaisante la réalité perçue, que résident les grands défis scientifiques. Tant que de tels «mystères» subsistent, la question de savoir si ces questions peuvent être résolues avec les instruments usuels de la science restera ouverte.

Bruno Kesseli 